Revue d'histoire de l'Amérique française

BEVUE D.HISTOIRE DE L'AMÉRIQUE FRANÇAISE

\title{
RAYSIDE, David M., A Small Town in Modem Times: Alexandria, Ontario. Kingston et Montréal, McGill-Queen's University Press, 1991. 336 p.
}

\section{Odette Vincent-Domey}

Volume 45, numéro 3, hiver 1992

URI : https://id.erudit.org/iderudit/305007ar

DOI : https://doi.org/10.7202/305007ar

Aller au sommaire du numéro

Éditeur(s)

Institut d'histoire de l'Amérique française

ISSN

0035-2357 (imprimé)

1492-1383 (numérique)

Découvrir la revue

Citer ce compte rendu

Vincent-Domey, O. (1992). Compte rendu de [RAYSIDE, David M., A Small Town in Modem Times: Alexandria, Ontario. Kingston et Montréal, McGill-Queen's University Press, 1991. 336 p.] Revue d'histoire de l'Amérique française, 45(3), 462-464. https://doi.org/10.7202/305007ar d'utilisation que vous pouvez consulter en ligne.

https://apropos.erudit.org/fr/usagers/politique-dutilisation/ 
RAYSIDE, David M., A Small Town in Modern Times: Alexandria, Ontario. Kingston et Montréal, McGill-Queen's University Press, 1991. 336 p.

Bien que le titre de l'ouvrage du professeur Rayside annonce une monographie locale bien campée dans le temps et l'espace, l'ampleur des objectifs visés par l'auteur et la méthodologie utilisée transcendent volontiers le cadre étroit de la monographie locale descriptive. Avec des moyens modestes, selon les termes de l'auteur, cette recherche s'inscrit dans la tradition sociologique des community studies qui se sont multipliées au Canada et aux États-Unis depuis la fin des années 1950. Inspirée par des préoccupations tout à fait contemporaines, cette étude globale d'une «petite» ville ontarienne, bilingue et biculturelle, aborde avec une sensibilité raffinée les domaines plus vastes des relations entre les classes sociales, les ethnies et les sexes [genders] dans la ville d'Alexandria, comté de Glengarry. D'abord tenté d'étudier le comté de Glengarry dans son ensemble, l'auteur se ravisa en cours de recherche pour se concentrer sur son principal centre urbain, Alexandria [3 246 habitants en 1986], perçu comme un microcosme de la société canadienne.

L'originalité de l'étude repose avant tout sur le contraste, souligné tout au long de l'ouvrage, entre, d'une part, la perception qu'ont les citoyens de leur ville en tant que communauté fraternelle et, d'autre part, l'existence d'inégalités sociales persistantes construites dans le temps entre les différents groupes sociaux et ethniques. Le premier chapitre, «The Lie of the Land», donne le ton général: en voulant aller au-delà de l'image complaisante de la municipalité reflétée dans le journal local, le Glengarry News, l'auteur cherche à comprendre les relations complexes entre les acteurs économiques, sociaux et politiques au sein de la petite ville, dans l'espoir d'y discerner une image plus conforme à la réalité des gens (p. xii). À cet égard, sa source principale consiste en un corpus de plus de 150 entrevues informelles avec des résidants, en majorité des gens ordinaires - low profile - de la municipalité. Appuyée sur une diversité d'autres sources documentaires, tels les recensements canadiens, et les rapports et dossiers des conseils scolaires et municipaux, l'étude traite de façon thématique les différents aspects de la vie de la municipalité. Au fil des chapitres, le développement industriel et économique, le monde du travail salarié, l'inégalité basée sur le sexe, la langue et le monde scolaire, la politique locale sont tour à tour abordés. Fidèle à son objectif avoué en début de volume de privilégier «le contact intime» (p. xiii) avec son objet d'étude, l'auteur conclut inévitablement sur un paradoxe. Fragmentée, la société d'Alexandria l'est bien davantage que ne le suggère l'idée que s'en font la majorité de ses habitants. Mais en même temps, on retrouve parmi sa population une cohésion plus importante que dans une grande ville.

L'auteur aborde chacun des thèmes privilégiés sous l'angle de l'impact du monde extérieur - entendre les pouvoirs politiques et économiques des grands centres - sur la petite communauté sociale. Le fait que les changements soient provoqués de l'extérieur contribue à expliquer en grande partie l'apparente quiétude - quiescence - de la vie politique locale. En 
partie seulement car, selon l'auteur, l'absence de contrôle des pouvoirs locaux sur les décisions majeures affectant la vie de la communauté locale de même que la montée du rôle des experts — de l'extérieur — n'ont pas complètement immobilisé la population locale qui a tout de même réussi à s'organiser en syndicats dans les entreprises de la région et à mener à terme certains projets sociaux d'abord froidement reçus par les élus municipaux.

Les disparités sociales camouflées sous la réputation d'une petite ville tranquille et harmonieuse éclatent au grand jour dans les chapitres consacrés aux rapports entre les sexes et aux questions linguistiques. Sur les inégalités entre les hommes et les femmes, rien de nouveau si ce n'est la constatation d'une perception plus traditionnelle des rôles dans ce milieu étant donné la faible diversification des emplois féminins accessibles et l'importance de la famille en tant que réseau social (p. 135-145). Cette partie de l'analyse concernant la famille et son influence n'est toutefois pas entièrement convaincante. L'analyse est ici moins nuancée que dans les autres chapitres. À l'encontre des conclusions de nombreuses études historiques sur la famille, on a l'impression (p. 291) que, pour l'auteur, la famille nucléaire est une invention toute récente. L'importance qu'il attribue aux traditions pèse trop lourdement dans le phénomène de la reproduction sociale, en contradiction flagrante avec l'évolution du système scolaire. Sans nier l'ampleur du phénomène, on peut douter que, dans les années 1980, la tendance des enfants de la classe ouvrière à restreindre leur choix d'avenir aux seuls métiers manuels soit aussi généralisée que le prétend Rayside (p. 74). Il serait étonnant que, même à Alexandria, cette attitude soit si prononcée.

«The Distinct Worlds of Anglophones, Francophones and Bilinguals» en fera peut-être sursauter plusieurs: la société distincte est un concept fort malmené dans la période actuelle! Ce chapitre consacré à la question linguistique constitue, à notre avis, un point fort de l'étude de Rayside. Toute en nuance, l'approche fait ressortir comment «the burden of accomodating to two language groups within a single community falls upon French speakers» (p. 178). Même si l'auteur constate qu'il y a plus de contact entre les deux groupes linguistiques à Alexandria qu'ailleurs au Canada, il remarque également que les deux communautés ont tendance à se replier sur leurs propres institutions - le groupe des bilingues s'accommodant davantage des deux réseaux. Cette situation, conjuguée aux décisions gouvernementales provinciales dans le secteur de l'éducation, a mené à une crise scolaire fort justement analysée dans le chapitre 5, «Language and Schooling», où les arguments des uns et des autres sont pertinemment remis en contexte.

En ce qui concerne la présentation matérielle, on ne peut que déplorer la piètre qualité des cartes géographiques. Étant donné l'importance des pouvoirs économiques et politiques extérieurs dans la problématique générale du volume, la carte d'introduction - qui ne couvre que le tiers supérieur de la page - aurait gagné à être plus lisible. Montréal, Ottawa, Cornwall cachée sous la route 401 - de même que Toronto devraient y apparaître plus clairement. Au chapitre 1, la description fragmentaire de la ville, qui est entrecoupée d'extraits d'entrevues, complique le repérage physique et renvoie impérieusement à la carte artisanale de la ville qui suit la préface. 
Somme toute, il s'agit là d'un ouvrage fort bien documenté, qui tout en s'adressant à plusieurs publics, rejoint dans ses objectifs les grandes questions de l'histoire sociale actuelle: les rapports entre les sexes, les groupes ethniques et les classes sociales. Malgré quelques faiblesses, notamment au niveau de l'analyse historique de la famille, le volume contribue de façon originale à notre connaissance des «petits» centres qui constituent un maillon important de la trame urbaine canadienne.

Institut québécois de recherche

sur la culture

ODETTE VINCENT-DOMEY 